\title{
Arrhythmogenic right ventricular cardiomyopathy in a 52-year-old man - clinical presentation mimicking an acute coronary syndrome
}

\author{
Łukasz Zandecki, Marcin Sadowski, Jacek Kurzawski, Agnieszka Janion-Sadowska \\ Świętokrzyskie Cardiology Center, Provincial Hospital Complex, Kielce, Poland
}

Postep Kardiol Inter 2013; 9, 3 (33): 256-261

DOI: $10.5114 /$ pwki.2013.37505

\begin{abstract}
Arrhythmogenic right ventricular cardiomyopathy is an infrequently diagnosed, genetically determined disease that leads to significant clinical consequences, including progressive heart failure and ventricular arrhythmias accounting for sudden cardiac death. We report the case of a 52-year-old patient who presented with ventricular tachycardia and features of an acute coronary syndrome. However, routine tests excluded critical coronary stenosis and the final diagnosis was arrhythmogenic right ventricular cardiomyopathy.
\end{abstract}

Key words: arrhythmogenic right ventricular cardiomyopathy, acute coronary syndrome.

\section{Introduction}

Arrhythmogenic right ventricular cardiomyopathy (ARVC) is a rare genetic disease in which the right heart muscle is replaced by fatty and fibrous tissue. The disease has significant clinical consequences including progressive heart failure and ventricular arrhythmias accounting for sudden cardiac death. Although diagnostic criteria of ARVC have been established, the prompt and accurate diagnosis may sometimes be a challenge. The initial symptoms usually include palpitations, syncope and even sudden cardiac death. However, it is not rare for the first manifestation of ARVC to mimic an acute coronary syndrome, particularly when concomitant left ventricular damage is present.

We report the case of a 52-year-old patient who presented with ventricular tachycardia and features of an acute coronary syndrome. Routine tests excluded critical coronary stenosis and the patient was finally diagnosed with ARVC.

\section{Case report}

This 52-year-old man, untreated for any chronic disease, was admitted to the hospital with palpitations, weakness and chest pain. The symptoms developed suddenly in the afternoon during routine activity. On admission the patient was in a serious condition, with clammy moist skin, blood pressure $80 / 50 \mathrm{~mm} \mathrm{Hg}$, regular heart rhythm of $180 \mathrm{bpm}$. The ECG revealed wide QRS tachycardia with left bundle branch block (LBBB) morphology (Figure 1). Because of hemodynamic instability cardioversion was performed using a 70 Joules DC shock with restoration of sinus rhythm at $55 \mathrm{bpm}$. Further evaluation revealed sinus bradycardia with dynamic changes in the ST-T segment (Figures 2 and 3).

An echocardiogram showed discrete hypokinesis of the distal inferolateral and anterolateral wall with preserved left ventricular ejection fraction (EF 63\%). There was a significant dynamic increase followed by a decrease in myocardial necrosis markers: troponin T hs - 365...753...643 ng/l, CK-MB $32 . .37 . . .49 \mathrm{U} / \mathrm{l}$, and increased activity of aminotransferase (ALAT $253 \mathrm{U} / \mathrm{l}$, AST $260 \mathrm{U} / \mathrm{l}$ ) and D-dimers (1616 $\mu \mathrm{g} / \mathrm{l})$.

Given the clinical presentation, ECG recordings and changes in markers of myocardial injury, a preliminary diagnosis of acute coronary syndrome (ACS) was made. Urgent coronary angiography revealed diffuse coronary artery lumen irregularities which appeared hemodynamically insignificant (narrowing to about 30-40\%).

Transthoracic and transesophageal echocardiography showed an enlarged (40-55 mm) right ventricular cavity with abnormal moderator band and free wall thinning below the tricuspid annulus and in the apical segment (Figure 4). Leftto-right shunt at the atrial level was excluded.

As the echocardiograms and ventricular tachycardia (VT) episode raised suspicion of ARVC an magnetic resonance imaging (MRI) scan was obtained which revealed the typ- 
ical features of ARVC (Figures 5). Continuous ECG recording showed sinus bradycardia during the day up to 37-40 bpm (maximal rate $71 \mathrm{bpm}$, mean $54 \mathrm{bpm}$ ) with numerous ventricular escape beats about $50 \mathrm{bpm}$.

Taking into account all signs and symptoms the patient was finally diagnosed as having ARVC with hemodynamically significant ventricular tachycardia, sick sinus syndrome requiring permanent pacemaker implantation and stable coronary artery disease.

The elevation in markers of myocardial injury was ascribed to the episode of ventricular tachycardia and cardioversion, whereas segmental wall motion abnormalities were ascribed to the involvement of the left ventricle with the disease process, which was confirmed by MRI scanning. Changes in the ST-T segment after the episode of ventricular tachycardia could be a result of transient myocardial ischemia due to the imbalance between increased myocardial oxygen demand and decreased coronary blood flow during VT. In fact the ST segment depression during and after the VT episode is not a rare occurrence and does not need to indicate acute myocardial ischemia whereas inverted T waves in chest leads V1-V3 are typical features of ARVC. In secondary prevention of sudden cardiac arrest and sudden cardiac death the patient received a dual chamber pacemaker.

\section{Discussion}

Arrhythmogenic right ventricular cardiomyopathy, also known as arrhythmogenic right ventricular dysplasia, is a rare genetic disease in which the right heart muscle, and later on also the left heart muscle, is replaced by fatty and fibrous tissue. The disease has significant clinical consequences including progressive heart failure and ventricular arrhythmias which may cause sudden cardiac death (SCD).

The prevalence of ARVC in the general population is $1: 2000-1: 5000$, affecting men more frequently than women, and the first symptoms usually occur in young adults (on average at age 29) [1]. Reports on ARVC developing in people $>50$ years of age are infrequent. It is estimated that $15-25 \%$ of sudden cardiac deaths are caused by ARVC, and the annual rate of SCD among patients with ARVC is $2 \%[2]$.

The first symptom of ARVC is usually an irregular heart beat (palpitations) or syncope in $27 \%$ and $26 \%$ of patients, respectively. Unfortunately, sudden cardiac death is the first clinical manifestation of the disease in $23 \%$ of cases [1]. The most typical clinical sign of ARVC is symptomatic right ventricular arrhythmia with left bundle branch block morphology developing either ventricular extrasystoles or sustained tachycardia that may degenerate to ventricular fibrillation. Arrhythmias in patients with ARVC are most frequently induced by physical activity. Moreover, long-term vigorous physical activity leads to right ventricular volume overload. It may result in disruption of desmosomal adhesion in the right ventricular wall and trigger the cascade of events lead-

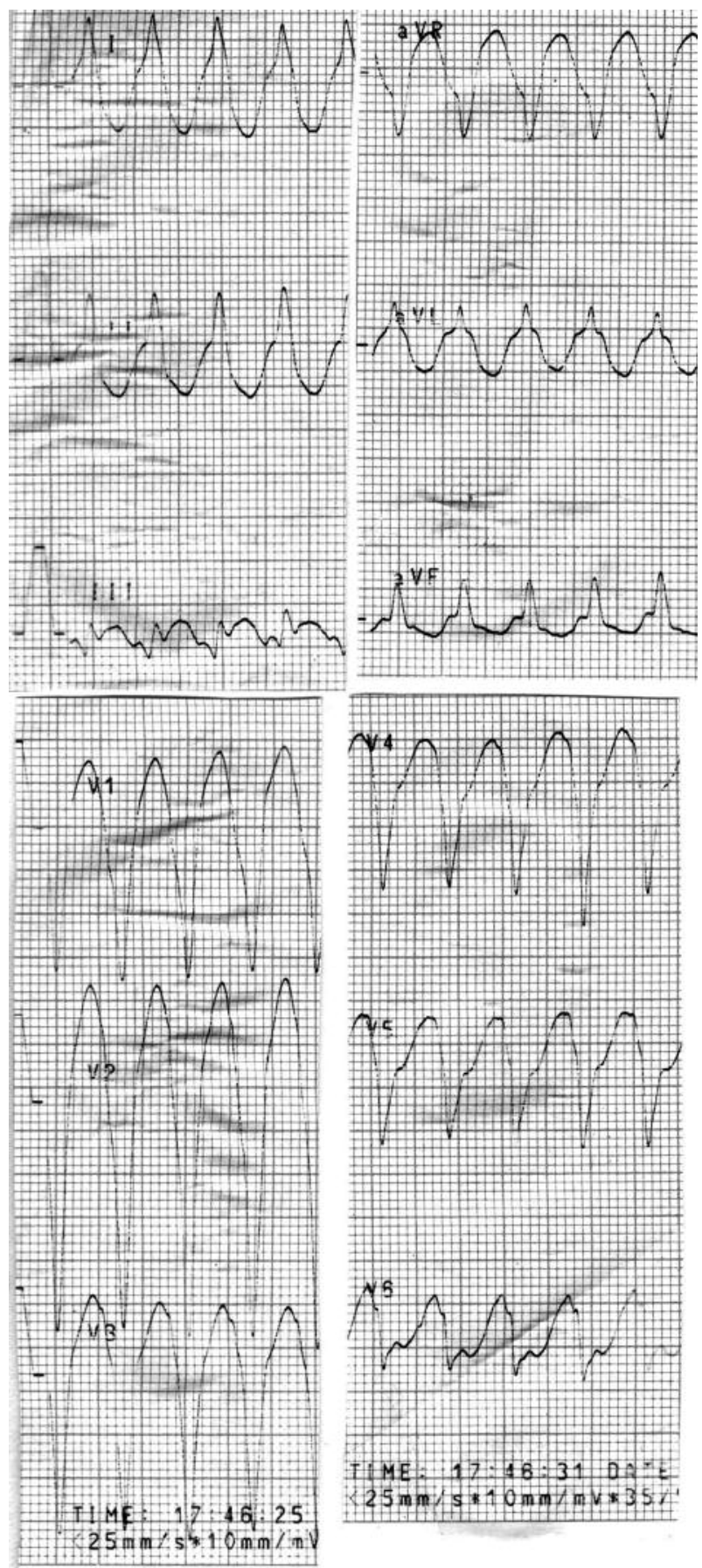

Fig. 1. Ventricular tachycardia of $180 \mathrm{bpm}$ with left bundle branch block morphology and inferior electrical heart axis

ing to myocardial remodeling and disease progression. For this reason it is important to advise patients with ARVC to avoid competitive sport [3].

No single diagnostic technique is sufficiently sensitive and specific to diagnose ARVC. Magnetic resonance imaging, believed so far to be the unquestionable test, produces false positive results. Endomyocardial biopsy neither excludes (the biopsy samples may come from the segments that are not involved in the disease process) nor confirms 


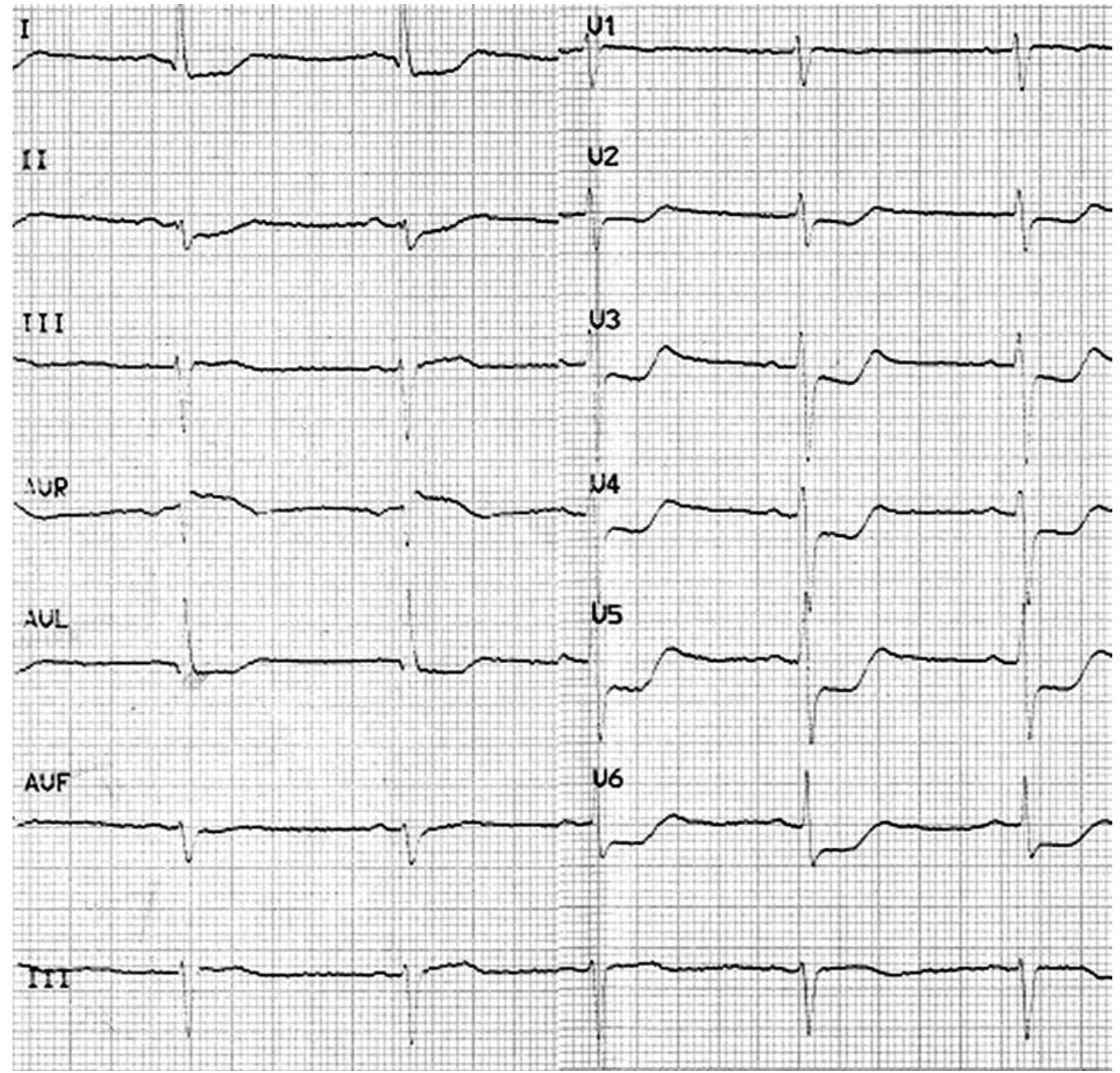

Fig. 2. ECG immediately after DC cardioversion: pathological left axis deviation. Sinus rhythm of $60 \mathrm{bpm}$. ST segment depression of 2-4 $\mathrm{mm}$ in lead I, AVL, V2-V6 with biphasic T waves representing myocardial ischemia. ST segment elevation in lead AVR

the condition (small lesions of this type may also be seen in healthy subjects); moreover, it is an invasive procedure with a risk of serious complications. In 1994 the ESC and ISFC Working Group on Myocardial and Pericardial Diseases established diagnostic criteria of ARVC. These were based on imaging tests (echocardiography, MRI or ventriculography), ECG at rest and continuous ECG recording, history of syncope, episodes of VT and VF, familial disease and endomyocardial biopsy. In order to improve diagnostic sensitivity as well as to incorporate new technology and advances in genetics, modification and update of the first guidelines was proposed 16 years after the first publication [4]. The diagnosis of ARVC is fulfilled by the presence of two major or one major and two minor or four minor criteria from different categories. In the present report two major and one minor criteria were fulfilled: right ventricular end-diastolic volume index (RVED/BSA) assessed by MRI was $126 \mathrm{ml} / \mathrm{m}^{2}$ (major criterion), inverted T waves in leads V1, V2 and V3 without the presence of right bundle branch block (RBBB) (major criterion) and documented sustained ventricular tachycardia of LBBB morphology with an inferior axis (minor criterion).

Arrhythmogenic right ventricular cardiomyopathy is a genetically determined disorder associated with autosomal dominant inheritance in over $30 \%$ of cases with incomplete penetration and variable expression. Recessive variants are less frequent and usually associated with more severe forms of the disease. So far a few dozen genes have been iden- 


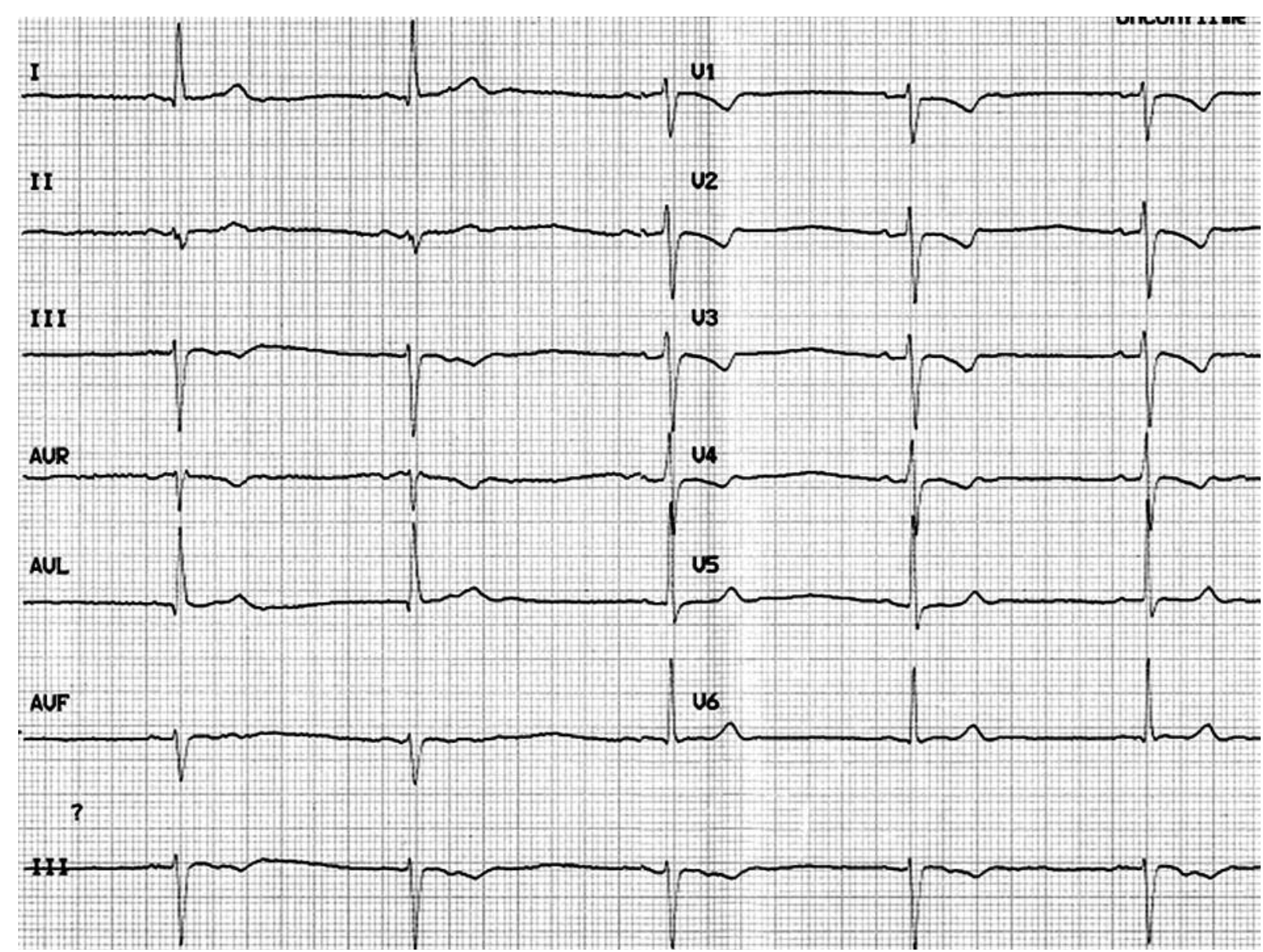

Fig. 3. ECG during hospitalization: pathological left axis deviation. Sinus rhythm of $47 \mathrm{bpm}$. Negative T waves in leads V1-V4, flat and negative in lead III, AVF

tified that are associated with ARVC and several others need to be verified. Mutations in genes encoding desmosomal proteins maintaining the structural integrity and cell signaling are most important [2]. Genetic testing may have a valuable role in confirming the diagnosis of ARVC in a proband (as one major criterion) and detecting the disease among family members before the first symptoms appear. It is also possible to provide them with genetic counseling to monitor the development of the disease and to assess the risk of transmitting the disease to offspring [5].

Electrocardiography abnormalities often reveal signs of ARVC. Inverted T waves in leads V1-V3 without RBBB are seen relatively frequently (in $85 \%$ of patients) [1]. Such ECG changes were also found in our patient. However, in some patients (14-18\%) QRS complexes have RBBB-like morphology. In fact, usually it is not RBBB but an intraventricular block resulting from slowed conduction in abnormal tissue of the right ventricle. For the same reason slow and inhomogeneous repolarization results in epsilon waves and late potentials. The recording and identification of epsilon waves may be difficult. Reduced paper speed, amplification or placing the right ventricular leads (V1-V3) one intercostal space higher than for standard ECG recording may turn out useful [6]. Multiple ECG recordings in our patient did not show epsilon waves.

Echocardiography usually reveals right ventricular structural abnormalities, but we should remember that ECG changes and arrhythmias may be present before myocyte degeneration in histology and before clinical and echocardiographic signs of RV dysfunction [1].

Patients with ARVC may also suffer from coronary artery disease, similar to our patient, and ventricular tachycardia may be a manifestation of an acute coronary syndrome. In fact, acute ischemia of the right ventricle in ARVC patients can easily induce VT. The differentiation of VT in the course of ARVC without acute ischemia from VT in the course of acute coronary syndrome may be difficult, especially in the presence of typical chest pain, new wall motion abnormalities on echocardiography or significant dynamic rise in markers of myocardial necrosis. Similar to other investigators reporting on ARVC mimicking ACS $[6,7]$, we decided to perform coronary angiography as the first choice to exclude acute coronary occlusion, especially as it was the first clinical manifestation of ARVC.

Implantation of a cardioverter-defibrillator is a highly effective and recommended method to prevent SCD in high- 


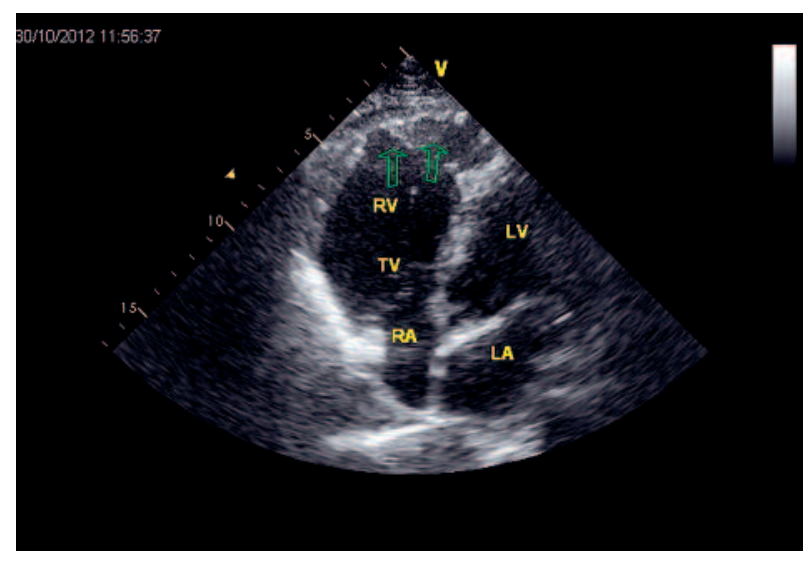

Fig. 4. Transthoracic echocardiogram. Modified 4-chamber view: enlarged $(40-55 \mathrm{~mm})$ right ventricular cavity with the thickened moderator band and free right ventricular wall thinning below the tricuspid annulus and in the apical segment

risk patients with ARVC. It should be however remembered that such patients are especially prone to procedure-related complications and reoperations which are not infrequent considering the relatively young age at diagnosis and progressive character of the disease. Increased procedure-related risk is associated with the fact that fibrofatty changes in the right ventricle may interfere with adequate lead positioning, and thin RV walls increase the risk of perforation [8]. latrogenic (lead-related) tricuspid regurgitation further deteriorates the function of the abnormal right ventricle [9]. Moreover, dynamic changes in the structure and electrophysiology of the right ventricle in patients with ARVC can hamper detection of arrhythmias and result in pronounced increases in pacing and defibrillation thresholds [1].

Taking into account the relatively young age at diagnosis and possible decision to implant an ICD, as well as complications and difficulties with endocavitary electrode placement, subcutaneous ICDS (S-ICD) appear to be a promising new alternative for patients with ARVC. It is however important to note that S-ICDs do not provide antitachycardia pacing (ATP), which in a sense limits their use in patients with VT episodes. Our patient additionally had sick sinus syndrome resulting in profound sinus bradycardia requiring permanent pacing, which cannot be provided by S-ICDs.

Risk stratification is an important issue that determines the management of ARVC. Patients with a history of sudden cardiac arrest, unstable VT and syncope are at the highest risk of arrhythmic events. Stable VT, early ( $<35$ years of age) onset of the disease and extensive myocardial involvement appear to be associated with at least moderate risk. It is still unclear whether genetic testing and results of electrophysiological studies have an impact on risk assessment [2]

In accordance with the ESC 2006 Guidelines ICD implantation carries a class I recommendation for patients with ARVC who have documented sustained VT or VF and are receiving long-term optimal pharmacotherapy [5].

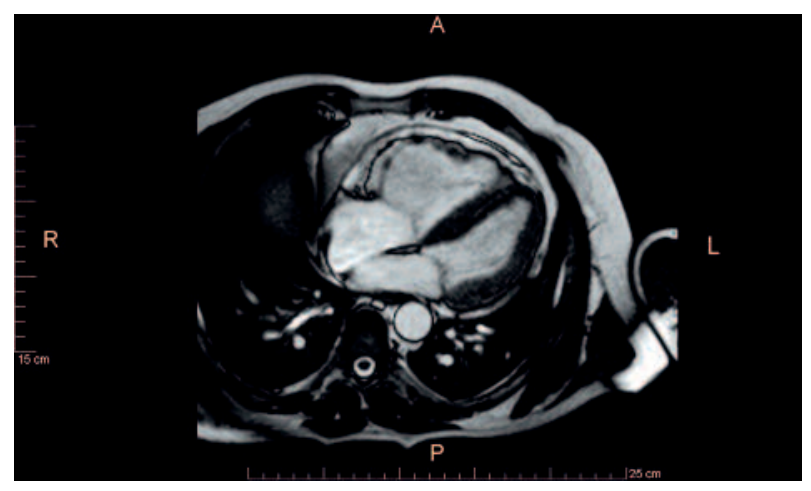

Fig. 5. MRI: extensive fat infiltration of the enlarged right ventricular free wall. Right ventricular end-diastolic volume index (RVED/BSA) of $126 \mathrm{ml} / \mathrm{m}^{2}$

As our patient with ARVC and an episode of VT had profound sinus bradycardia with multiple ectopic beats, we decided to implant a dual chamber cardioverter-defibrillator, taking into account prospective pharmacotherapy. Furthermore, possible application of dual chamber arrhythmia detection algorithms reduces the frequency of inadequate ICD discharges triggered by supraventricular tachyarrhythmias, which are relatively frequent in such patients [1].

The treatment of ARVC focuses on avoiding heart failure and arrhythmia. Sotalol, usually in high doses (320-640 mg daily), is the most efficacious antiarrhythmic drug [3]. If sotalol is not effective, other antiarrhythmic drugs are unlikely to be of benefit, especially in patients with inducible VT. In patients with non-inducible VT, $\beta$-blockers and verapamil may be used as an alternative treatment [1].

In patients with recurrent VT despite optimal antiarrhythmic drug therapy, catheter ablation may be considered as adjunctive treatment [5]. Studies provide conflicting results on the effectiveness of ablation. Short-term efficacy is 5090\% depending on the technique, operator's experience and individual patient characteristics. However, during natural progression of the disease recurrent arrhythmias are quite common. In patients with ARVC arrhythmogenic foci are frequently located not only in the endocardium, but also in the epicardium or within the myocardial walls. For this reason, epicardial mapping and ablation may provide a better outcome for these patients [9].

Orthotopic heart transplantation is the only option providing a complete cure. However, due to limited availability it is reserved for a selected small group of patients with end-stage heart failure and drug-resistant ventricular tachyarrhythmias [8].

\section{References}

1. Azaouagh A, Churzidse S, Konorza T, et al. Arrhythmogenic right ventricular cardiomyopathy/dysplasia: a review and update. Clin Res Cardiol 2011; 100: 383-394.

2. Paul M, Wichter T, Fabritz L, et al. Arrhythmogenic right ventricular cardiomyopathy: an update on pathophysiology, genetics, diagnosis, and risk stratification. Herzschrittmacherther Elektrophysiol 2012; 23: 186-195. 
3. Woźniak O, Biernacka EK. Prewencja nagłych zgonów w arytmogennej kardiomiopatii prawej komory - jak oceniać ryzyko i kiedy wszczepić pacjentowi kardiowerter-defibrylator? Folia Cardiologica Excerpta 2010; 5: 27-30.

4. Marcus FI, McKenna WJ, Sherrill D, et al. Diagnosis of arrhythmogenic right ventricular cardiomyopathy/dysplasia: proposed modification of the Task Force Criteria. Eur Heart J 2010; 31: 806-814.

5. Zipes DP, Camm AJ, Borggrefe M, et al. ACC/AHA/ESC 2006 Guidelines for Management of Patients With Ventricular Arrhythmias and the Prevention of Sudden Cardiac Death: a report of the American College of Cardiology/American Heart Association Task Force and the European Society of Cardiology Committee for Practice Guidelines (writing committee to develop Guidelines for Management of Patients With Ventricular Arrhythmias and the Prevention of Sudden Cardiac Death): developed in collaboration with the European Heart Rhythm Association and the Heart Rhythm Society. Circulation 2006; 114: e385-e484.

6. Kukla P, Biernacka EK, Jastrzębski M, et al. Arrhythmogenic right ventricular cardiomyopathy with left ventricular involvement mimicking acute coronary syndrome - two case reports. Kardiol Pol 2011; 69: 470-474.

7. Khalil SI, Kamal A, Ahmad S. Arrhythmogenic right ventricular dysplasia presenting as acute coronary syndrome: a case report. Eur J Echocardiogr 2004; 5: 394-398.

8. Peters MN, Katz MJ, Alkadri ME. Diagnosis of arrhythmogenic right ventricular cardiomyopathy. Proc (Bayl Univ Med Cent) 2012; 25: 349-353.

9. Arbelo E, Josephson ME. Ablation of ventricular arrhythmias in arrhythmogenic right ventricular dysplasia. J Cardiovasc Electrophysiol 2010; 21: 473-486. 